\title{
ZUR ENTWICKLUNG EINER NEUEN PÄDAGOGISCHEN WISSENSCHAFTSORIENTIERUNG IN MITTEL- UND OSTEUROPA UND DEREN UMSETZUNG IN DIE PÄDAGOGISCHE PRAXIS ${ }^{1}$
}

\section{Zusammenfassung}

In einem ersten Teil diskutiert der Beitrag die Frage von sog. ,fortschrittlichen' pädagogischen Neuerungen und Veränderungen, die allerdings nur überzeugend wirken können, wenn sie auf einer sicheren Argumentations- und Legitimationsbasis beruhen. Wir müssen uns dagegen verwahren, dass in der Pädagogik der ,Mode` angepasstes Gedankengut zu Ideen, ja Fiktionen hochstilisiert wird, das in seinen logischen Konsequenzen eher der Verwirrung der Strukturierungen und Fachsprache diene, wie Franz Hofmann ${ }^{2}$ bereits anmahnte. Erkenntnisse aus der Wissenschaftsgeschichte sind Ausgangspunkt der Überlegungen.

Der zweite Teil befasst sich mit der praktischen Konsequenz, dem Zusammenhang von Unterrichtsweise und persönlicher Einstellung der Lehrenden und den damit verbundenen Auswirkungen auf das Lernklima. Es werden wesentliche Defizite gegenwärtiger Arbeit angesprochen, und es wird versucht, über das ,Gegenbild' von Unterricht in Form einer überkommenen, problematischen Auffassung von Unterrichten, einen vom aktuellen Lehrerethos ge-

\footnotetext{
${ }^{1}$ Der Beitrag stellt eine stark gekürzte und veränderte Fassung folgenden Beitrags dar: Carlsburg, G.-B. von: Neuorientierung von Enkulturation und Bildung in Mittel- und Osteuropa. In: Carlsburg, G.-B. von (Hrsg.). Enkulturation und Bildung. Frankfurt a.M.: Peter Lang, 2009, S. 265-281.

Die Idee zu dieser Thematik entstand aus der Zusammenarbeit mit dem verstorbenen Dresdener Emeritus Gerhard Arnhardt, Schüler des verstorbenen Haller Emeritus' Franz Hofmann, sowie aus meinen Reisen in die MOE-Staaten und Russland ab 1993. Als MOE-Staaten seien hier insbesondere folgende Staaten in die Diskussion einbezogen: Litauen, Estland, Lettland, Polen, Tschechien, Slowakei, Russland, Ukraine, Weißrussland, ebenso treffen diese Rückschlüsse auch auf die ehemalige Deutsche Demokratische Republik zu.

2 Hofmann, F. Probleme der "Allgemeinen Pädagogik“ als Aufgabe der pädagogischen Grundlagenforschung. In Hofmann, F., Fritzke, Chr. (Hrsg.): „Allgemeine Pädagogik“ als Anliegen pädagogischer Grundlagenforschung. Kongreß- und Tagungsberichte (KTB) der Martin-Luther-Universität HalleWittenberg (MLU). Wissenschaftliche Beiträge 26 (E 23). Halle: MLU, 1979, S. 5-33, insbes. S. 5.
}

prägten Unterricht auf der Grundlage historisch begründeter und dem Zeitgeist naher Sach- und Handlungskompetenzen vorzustellen.

\section{Abstract}

In the first part discusses the contribution the question of so-called 'progressive' educational innovations and changes that can only work, however convincing, if they are based on a secure basis of legitimacy and argumentation. We must protest against that in education, 'fashion' adapted ideology to ideas, even fiction is highly stylized, which is used in its logical consequences rather the confusion in the structuring and specialized language, such as Franz Hofmann $^{2}$ already sends a reminder. Knowledge from the history of science is the starting point of our deliberations.

The second part focuses with the practical consequence, the relationship between teaching method and personal settings of the teachers and the associated impact on the learning environment. It addresses significantly shortcomings of current work, and tries to imagine about that 'antitype' of teaching in the form of a traditional, problematic conception of teaching, one of the current teacher ethos characterized teaching on the basis of historically grounded, and the times soon technical and professional competences.

\section{Schlüsselwörter}

Bildungsprozesse, Wissenschaftsgeschichte, Wissenschaftssprache, Normen- und Wertediskussion, Neuorientierung der Pädagogik, handlungsorientierter Unterricht, ,Gegenbild' von Unterricht, Lernklima, lebenslanges Lernen (LLL), Lehrerethos, Sach- und Handlungskompetenzen, Unterrichtsalternativen.

\section{Vorbemerkung}

Durch den aktiven Einbezug mittel- und osteuropäischer Wissenschaftler in die erziehungswissen- 
schaftliche Diskussion und dem damit verbundenen persönlichen Austausch von Forschungsergebnissen seit 1990 hat die Komparatistik in Deutschland eine neue Qualität erhalten. Insbesondere dem Frankfurter Emeritus Wolfgang Mitter (geb. 1927), 19781981 und 1987-1995 Direktor des DIPF (Deutsches Institut für Internationale Pädagogische Forschung) in Frankfurt, war es seit den sechziger Jahren vorbehalten, vergleichende Forschungen in der Sowjetunion durchzuführen. Er galt lange Zeit zusammen mit dem Bochumer Emeritus Oskar Anweiler (geb. 1925) als ausgewiesener Ostexperte. Die estnischen, litauischen und lettischen Wissenschaftler/-innen standen schon in der sowjetischen Zeit in engem Kontakt zu den nordeuropäischen Nachbarn, was seitens der Moskauer Zentrale toleriert wurde, nahmen doch die drei baltischen Sowjetrepubliken einen gewissen autonomen Status mit eigener Sprache innerhalb der Sowjetunion ein. Der Wunsch der baltischen Völker nach Integration in die Europäische Union fand am 1. Mai 2004 seine Erfüllung.

I. Aktuelle Aufgaben einer Neuorientierung der Pädagogik in den MOE-Staaten

In jeder Etappe der Neuformierung des pädagogischen Selbstbewusstseins war es eine wissenschaftliche Torheit, das Streben nach dem Allgemeinen in der Pädagogik in irgendeiner Art und Weise zu vernachlässigen. Hofmann machte bereits $1979^{3}$ darauf aufmerksam, dass die Pädagogik dann an Bedeutung gewann und erfolgreich war, wenn sie sich kontinuierlich der kritischen Auseinandersetzung mit der Erziehungspraxis stellte sowie das Ergebnis aus erziehungswissenschaftlicher Sicht philosophisch und wissenschaftstheoretisch zu analysieren wusste, in der Lage war, „Verstreutes oder Disparates systematisch zu verknüpfen" und sich insbesondere als zum Diskurs reizender Anwalt einer von der Vorgehensweise kritisch geleiteten „pädagogischen Vernunft" in der fortwährenden Auseinandersetzung mit dem sich laufend wandelnden Erziehungsalltag und der damit verbundenen Normen- und Wertediskussion zu erproben vermochte. ${ }^{4}$

Verwiesen werden soll an dieser Stelle insbesondere auf die Manipulationsversuche der russischen und sowjetischen Diktaturen, gegen die sowohl Leo Tolstoi ${ }^{5}$ als auch die russischen Publizisten, Schriftsteller und Pädagogen des 19. Jahrhunderts angegangen sind, insbesondere sei verwiesen auf den bekann-

\footnotetext{
${ }^{3}$ Ebd., S. 5-33.

${ }^{4}$ Ebd. S. 14

${ }^{5}$ Kegler, D. Lev Tolstoj: Ethizistische Pädagogik. In: Kegler, D. Das Ethos der russischen Pädagogik. Studien zum Erziehungsbegriff seit Pirogov. St. Augustin: Academia Verlag, 1991, S. 82-90.

ten Literaturkritiker und Kenner der Schellingschen und Hegelschen Philosophie Wissarion Grigorjewitsch Belinskij (1810-1848) noch Mitarbeiter der liberalen Zeitschrift „Der Zeitgenosse“, sowie an die Redakteure dieses Blattes, Nikolai Gawrilowitsch Tschernyschewskij (1828-1889) und dessen schon im Alter von 25 Jahren 1861 verstorbenen ,Schüler' Nikolai Alexandrowitsch Dobroljubow $^{7}$, die sich mit den Ideen Belinskijs und des Publizisten Alexander Iwanowitsch Herzen (18121870) ${ }^{8}$, dessen Namen die Pädagogische Universität St. Petersburg trägt, identifizierten.

Ihre Kritik bezog sich auf das zaristische Bildungssystem und Ihre Gedanken wurden richtungweisend sowohl für das Denken der , liberalen' russischen Demokraten im Sinne einer Erneuerung und Humanisierung des russischen Schulwesens bis zu Beginn der Stalin-Doktrin, wo diese Demokratisierungsbewegung abrupt abgebrochen wurde. Die Tradierung dieses Gedankenguts wurde wiederum bei den verantwortungsbewussten Pädagogen in der Zeit der Perestroika in der Sowjetunion aufgenommen, einschließlich der von ihr okkupierten Gebiete, wie beispielsweise die baltischen Staaten. Ähnliches kann man in den damaligen Ostblockstaaten registrieren.

Aber auch in demokratischen pädagogischen Bestrebungen stand der selbstauferlegte Zwang, mit einem Minimum an Zeit und Kraft, mit sog. ,griffigen Modeantworten', pädagogische Tagesfragen zu finden und ad hoc zur Verfügung zu stellen, historischen Erfahrungen entgegen. Anstatt als Konsequenz aus diesem Dilemma auf längere Sicht theoretische Anstrengungen im Grundlagenbereich zu leisten, wurde die theoriegeleitete Praxeologie $e^{9}$ oft mit vorschnellen verkürzten Anschauungen und Handlungen überdeckt, die praxiswirksam sein

\footnotetext{
${ }^{6}$ Vgl. Brockhaus' Konversations-Lexikon. Band 3. Leipzig/Berlin/ Wien: Brockhaus Verlag, ${ }^{14} 1894$; Brockhaus' Konversations-Lexikon. Band 5. Leipzig/Berlin/Wien: Brockhaus Verlag, ${ }^{14} 1894$; Brockhaus' Konversations-Lexikon. Band 9. Leipzig/Berlin/Wien: Brockhaus Verlag, ${ }^{14} 1894 ;$ Brockhaus' Konversations-Lexikon. Band 15. Leipzig/ Berlin/Wien: Brockhaus Verlag, ${ }^{14} 1895$; Belinski, W.G. Ausgewählte pädagogische Schriften. Berlin: Volk u. Wissen, 1953.

Vgl. Brockhaus' Konversations-Lexikon, Bde. 3/5/9, ebd.; Dobroljubow, N.A. Ausgewählte pädagogische Schriften. Berlin: Volk u. Wissen, 1956.

${ }^{8}$ Verwiesen wird hier auf den Kreis der „zapadniki“ [„Westlinge“] um Belinskij und Stankewitsch, wo Herzen mit der Hegelschen Philosophie vertraut gemacht wurde, und auf seine Moskauer publizistische Tätigkeit (1842-1847) unter dem Pseudonym Iskander. Danach ging er ins Ausland und gründete Exilblätter in London, später in Genf, vgl. Brockhaus' Konversations-Lexikon, Band 9, ebd., S. $101 \mathrm{f}$.

${ }^{9}$ Praxeologie im aristotelischen Sinne verstanden: als ethische Legitimierung menschlichen Handelns auf der Grundlage von Erkenntnis.
} 
sollten. Die Ausrichtung der Bildungssysteme auf direkte Berufsvorbereitung war und bleibt gegen das humanistische Grundanliegen gerichtet, Menschen allseitig auf das Leben vorzubereiten und auf der Grundlage einer soliden Allgemeinbildung Berufsentscheidungen herbeizuführen. ${ }^{10}$

Wenn wir nach Lösungsmöglichkeiten suchen, so müssen auch Hofmanns Ansätze ${ }^{11}$ zur Diskussion gestellt werden, Positionen, die bis heute kaum beachtet wurden und doch Probleme von aktueller Bedeutung skizzieren:

1. Die Bewältigung der Gegenwartsaufgaben zur Theorieentwicklung in der Bildung und Erziehung macht ein grundlegendes Verständnis der Wissenschaft unter historischem Einbezug zur Vorbedingung $^{12}$ Bei der damit verbundenen Auswahl weitreichender Konzeptionen, die inhaltlich repräsentativ und prägend über die Zeiten waren ${ }^{13}$, sind zu nennen:

I. Die "Didactica generalis", die allgemeine Lehrkunst des Jan Amos Comenius. Dieses Werk gehört zu den ersten umfassenden Versuchen, den allgemeinen philosophischen Ansprüchen an das Lehren und Lernen gerecht zu werden. In ihr bestimmt er die Ausformung der ,Ich-Identität' zur Triebkraft für das Allgemeine und leitet daraus verbindliche didaktische Konsequenzen ab. ${ }^{14}$.

II. Ein überzeitlicher Prüfstein für die Anregungskraft (Stimulans) der Bildungsgeschichte auf die Gestaltgebung der Pädagogik bleibt wohl Johann Heinrich Pestalozzis (1746-1827) Erziehungskonzeption mit der Fragestellung, inwiefern „Hilfe zur Selbsthilfe" gewährt werden kann und darf, wenn der Industriosität (Fleiß, Ethos, Ordnung, Exaktheit)

\footnotetext{
${ }^{10}$ Hierzu sei im Sinne der bereits erwähnten Tradierung auf die Kapitel VI und VII der „Große(n) Didaktik“, des Jan Amos Comenius (1592-1670) verwiesen: Comenius, J. A. Große Didaktik. VI. Kapitel. In: Arnhardt, G., Reinert [v. Carlsburg], G.-B. (Hrsg.). Jan Amos Comenius. Über sich und die Erneuerung von Wissenschaft, Erziehung und christlicher Lebensordnung. Bd. 2. Donauwörth: Auer Verlag, 1996, S. 381-395.

${ }^{11}$ Hofmann 1979, S. 5-33; Hofmann, F. Zum Erkenntniswert der Wissenschaftsgeschichte der Pädagogik. In Hofmann, F. (Hrsg.). Erkenntniswert einer Wissenschaftsgeschichte der Pädagogik. Kongreß- und Tagungsberichte (KTB) der MartinLuther-Universität Halle-Wittenberg (MLU). Wissenschaftliche Beiträge, Halle: MLU, 1980, 13 (E 28), S. 5-47; Hofmann, F. Wissenschaftssprache der Pädagogik in historischer Sicht. In Hofmann, F. (Hrsg.). Die Wissenschaftssprache der Pädagogik. Problemlogische und -historische Beiträge. Kongreß- und Tagungsberichte (KTB) der Martin-Luther-Universität HalleWittenberg (MLU), Wissenschaftliche Beiträge, Halle: MLU, 1985, 22 (E 65), S. 5-20; Hofmann, F. Studien zur Geschichte der bürgerlichen Didaktik. Berlin: Volk u. Wissen, 1989.

${ }^{12}$ Vgl. Hofmann 1979, S. 28 f.

${ }^{13}$ Vgl. ebd., S. 11

${ }^{14}$ Ebd., S. 11ff.
}

soziale Verwerfungen, bedingt durch den Zeitgeist (Sucht und Gier nach Reichtum und Macht) entgegenstehen. Hierbei wird von ihm die Kantische Philosophie, vermittelt durch Johann Gottlieb Fichte (1762-1814), verbunden mit einem tiefen inneren sozialpädagogischen Verantwortungsbewusstsein. ${ }^{15}$

III. Johann Friedrich Herbarts (1776-1841) „Allgemeine Pädagogik aus dem Zweck der Erziehung abgeleitet" $(1806)^{16}$ markiert das Paradigma des aus einer Kunst des Erziehens (,Erziehender Unterricht' als Basis des Zugangs von sittlicher Urteilskraft und Gewinnung von Autonomie) und psychologisch begründeten Allgemeinen, verbunden mit einem $\mathrm{Zu}$ gang für das Spekulative. In der Tradition der „Kritik der reinen Vernunft" Immanuel Kants ${ }^{17}$ nennt Hof$\operatorname{mann}^{18}$ diese Schrift eine vorwärtsweisende „Kritik der pädagogischen Vernunft ${ }^{\text {"19 }}$.

IV. Mit Friedrich Adolph Wilhelm Diesterweg (1790-1866) hatte sich der Dreiklang von Allgemeiner Pädagogik, Didaktik und Methodik formalisiert. Diese historisch gewachsene Trinität ist bis in unsere Zeit registrierbar, obwohl in Anbetracht aktueller insbesondere pädagogischer und lernpsychologischer - ,Wegweisungen' ein Teil des Gedankenguts nur noch tradierenden Charakter aufweist. ${ }^{20}$ Es handelte sich bei dem von F.A.W. Diesterweg 1835 bearbeiteten und herausgegebenen "Wegweiser zur Bildung für Lehrer und die Lehrer werden wollen, und methodisch-praktische Anweisungen zur Führung des Lehramtes”, ab 2. Auflage 1838 „Wegweiser zur Bildung für deutsche Lehrer" genannt, sowie in seiner 1820 verfassten Schrift „Ueber Erziehung im Allgemeinen und Schulerziehung im besonderen" ausschließlich um die Übergabe von Handlungshilfen im Lehrerseminar für die Bildung der Volksschullehrer. Die Klientel, die Adolph Diesterweg ansprach, verfügte nicht über die Grundlagen, Allgemeine Pä-

\footnotetext{
${ }^{15}$ Anführen ließe sich noch, dass die Reichweite im Hinblick auf die Zielgruppen von Burgdorf bis Iferten [Yverdon] sich veränderte. Aus diesem sozialpädagogischen Betreuungsimpetus entwickelte sich die reguläre Volksschule, als deren Begründer Pestalozzi firmiert. Die von ihm und seinen Mitarbeitern konzipierte Formalorganisation hat immer noch Bestand.

${ }^{16}$ Herbart, J. F.: Systematische Pädagogik. Eingel., ausgew. u. interpr. v. Benner, D. Stuttgart: Klett-Cotta, 1986, S. 71-191.

${ }_{17} 2$. verb. Aufl. 1787

${ }^{18}$ Ebd., 1979, S. 17.

${ }^{19} \mathrm{Vgl}$. Hofmann 1979, S. 13-17; 1980, S. 11ff.; 1989, S. 161ff.; vgl. auch Benner, D. (Hrsg.). Johann Friedrich Herbart. Systematische Pädagogik. Bände 1/2. Weinheim: Deutscher Studien Verlag, 1997; Herbart, J.F. Ausgewählte Schriften zur Pädagogik. Ausgew., eingel. u. erl. v. Hofmann, F. unter Mitarb. v. Ebert, B. Berlin: Volk u. Wissen, 1976, S. 67ff.

${ }^{20} \mathrm{Vgl}$. Hofmann 1979, S. 19 f., 1989, S. 197 ff.
} 
dagogik in dem hier definierten Bedeutungszusammenhang zu interpretieren. ${ }^{21}$

2. Um Einfluss auf die internationale Fortentwicklung einer wissenschaftsorientierten allgemeinen pädagogischen Theorie zu nehmen, ist als weitere wichtige Voraussetzung dem Einbezug und der Vervollkommnung der Wissenschafts- bzw. Fachsprache adäquate Bedeutung beizumessen. Alltags-, Fach-, Wissenschaftssprache aus antiken Ursprüngen und pädagogischer Terminologie der letzten Jahrzehnte müssen aus ihrer Mehrdeutigkeit zur Definition von grundlegenden Begriffen für die pädagogische Theorieentwicklung geführt werden; auszuschließen ist gleichzeitig die Tendenz zu trivialisierenden Allgemeinplätzen. ${ }^{22}$

Die Pädagogik ist gut beraten, stets die Nähe der allgemeinen Sprachentstehung und -entfaltung zu suchen, so wie es Johann Gottfried von Herder beispielsweise vermochte. Wesentliche Anstöße sind von ihm angeregt als auch umgesetzt worden. Das 1784 verfasste Kapitel „Slawische Völker“ der „Ideen zur Philosophie der Geschichte der Menschheit ${ }^{\text {“23 }}$, in welchem er für die im Umkreis „vom Don zur Elbe, von der Ostsee bis zum Adriatischen Meer“, „diesseit der karpatischen Gebürge, [...] jenseit derselben, wo sie frühe schon in der Walachei und Moldau saßen, "24 sich niederlassenden Völker Errettung aus der Sklaverei und „Erneuerung der alten Feste, des ruhigen Fleißes und Handels" ${ }^{\text {"25 }}$ ersehnte, erregte eigens bei der identitätssuchenden Jugend, „der jungen Intelligenz dieser Nationen", heftigen Nachhall. Herders Gedankengut, die große Anzahl der „Übersetzungen und Annotationen" seiner Ideen und Werke im Sprachraum der Slawen, sind Beweis seines Einflusses auf das Bildungs- und Erziehungswesen und -denken dieser Menschen ${ }^{26}$. Die mittel- und osteuropäische Geschichte zeigt, dass diese Länder nicht frei von Bestrebungen waren, die Herder bekämpfte.

3. Es muss dem Ausbau einer Systematik und „Binnenstruktur" einer wissenschaftlich fundierten Pädagogik mehr Beachtung geschenkt werden. Es lohnt der Mühe, tradierte Anstrengungen und Be-

\footnotetext{
${ }^{21} \mathrm{Vgl}$. Hofmann 1989, S. 197 ff.

22 Vgl. Hofmann 1979, S. 30f.; 1985, S. $5 \mathrm{ff}$

23 Herders Werke in fünf Bänden. Hrsg. von den Nationalen Forschungs- u. Gedenkstätten der klassischen deutschen Literatur in Weimar. Ausgew. u. eingel. v. Otto, R. Bibliothek Deutscher Klassiker. Band 4: Slawische Völker. 16. Buch. Kap. IV. Berlin/ Weimar: Aufbau-Verlag, ${ }^{5} 1978$, S. 393-400.

${ }^{24}$ Ebd., S. 393.

${ }^{25}$ Zit. Herder 1952 in Hofmann 1985, S. 17: Herder, J.G. Zur Philosophie der Geschichte. Eine Auswahl in zwei Bänden. Hrsg. von W. Harich. Zweiter Band. Reihe: Klassisches Erbe aus Philosophie und Geschichte. Berlin: Aufbau Verlag, 1952, S. 484 f.

${ }^{26}$ Zit. Herder, ebd. in Hofmann, ebd.

mühungen einer Systematisierung der Allgemeinen Pädagogik für die Lehrerbildung und Lehrerausbildung an internationalen Standards zu messen, um eine allseitig anerkannte Struktur von Theorien und Modellen einer zukünftigen Pädagogik zu entwickeln. Damit kann der Weg für eine weltweite $\mathrm{Zu}$ sammenarbeit beschritten werden. ${ }^{27}$

4. Pädagogik als Einheitswissenschaft ist bei ihrer „Filiation“ in Sub-Disziplinen zu hinterfragen, ob hinreichende Beachtung bei der Konstituierung von Untergliederungen den direkt damit zusammenhängenden „methodologischen“ Fragestellungen geschenkt wurde. ${ }^{28}$ Die Diskussion dazu ist in Deutschland kontrovers. Wesentlich ist, dass es nicht darum geht, ein für allemal Sub-Disziplinen festzulegen - und diese dann ausschließlich als gültig anzunehmen, denn jede Disziplin sollte sich, dem Erkenntnisfortschritt, dem Zeitgeist und den Bildungsnotwendigkeiten entsprechend, in ihrem eigenen Verständnis entwickeln, soweit dies goutiert wird, oder sie muss sich neu strukturieren, d.h. das deutsche klassifizierte Disziplindenken muss im Rahmen einer Globalisierung durch interdisziplinäres Denken aufgebrochen werden.

5. Viele Grundfragen des Bildungsinhalts und des pädagogischen Prozesses müssen auf ihre Praktikabilität überprüft werden. Das betrifft die latente Pädagogisierung von neuen Erkenntnissen genauso, wie die sozialpsychologische Fundierung des Prozessualen. Wesentliches der pädagogischen Theorieentwicklung muss einerseits ständig auf dem Prüfstand stehen, sich aber andererseits von den Erfordernissen des Tages abheben, wenn es sich nicht im Praktizismus erschöpfen oder sich selbst ad absurdum führen will. ${ }^{29}$ Das lehrt die vor allem sowjetisch geprägte Vergangenheit. Alle Versuche, dem Lehrer im Bildungs- und Erziehungsbereich allgemeinverbindliche Handlungsvorschriften in die Hand zu geben oder ihn darauf zu verpflichten, haben in das Dilemma eines manipulierten Lehrers geführt, der weder den Schülern die Möglichkeit zur Selbstverwirklichung, zum Lernen von Verantwortung im Unterricht gibt, noch sich selbst im Unterricht verwirklichen kann, weil er Übernahme von Verantwortung nicht gelernt hat. Unterrichtshilfen und Unterrichtsmaterialien, die im Detail bis zu Handlungsvorschriften für die einzelne Unterrichtsstunde gingen, standen dem Grundanliegen ,humanistischer Pädagogik entgegen, indem sie zwangsläufig mit und ohne Lehrerwillen auf Menschenmanipulation hinausliefen: so wur-

\footnotetext{
${ }^{27}$ Hofmann 1979, S. 31; vgl. 1980, S. 5 ff.

${ }^{28}$ Hofmann 1979, S. 31.

${ }^{29} \mathrm{Vgl}$. Ebd.
} 
den die Lehrsätze, selbst Tafelbilder etc. vorgegeben; so gab es in den sozialistischen Ländern Vorschriften in Form von Erziehungsplänen, von verbindlichen Erziehungszielen bis zu den Methoden, wie diese Ziele zu erreichen seien, unabhängig, losgelöst vom Schüler. ${ }^{30}$

6. Eine künftige Lehrerbildung sollte, wenn sie auf Dauer erfolgreich sein will, jener pädagogischen Konzeption weite Räume zugestehen, die pädagogische und literarische Innovation so in das Zentrum rückt, dass sie durch „Massenliteratur" und „-medien" gemeinsames Anliegen von Professionellem und Öffentlichem wird. Nur so kann Pädagogik als praktische Philosophie handlungsrelevant werden. ${ }^{31}$

Der gegenwärtigen Medienpädagogik, die ein Pflichtstudienbereich in der Lehrerausbildung sein sollte, muss in diesem Sinne eine bedeutende Bildungs- und Erziehungsfunktion zugewiesen werden. Sie kann nicht nur die neuen Lehr- und Lerntechnologien als Form der Ausbildung und des Unterrichtens nutzen, sondern sie sollte ihre Aufgaben als Mittler zwischen Individuum und Gesellschaft, vorrangig auf dem Sozialisations- und Enkulturationssektor wahrnehmen.

Sie sollte neue Technologien für die Erziehung durch Medien kanalisieren, auf schädigende Einflüsse, vor allem durch Gewaltdarstellungen in den Medien - vom Fernsehen über interaktives Video bis zum Computer - verweisen, diese durch gezielte Aufklärung und wirkungsvollen Boykott zu verhindern suchen, und aus entwicklungspsychologischer und anthropologischer Betrachtung letztlich eine wichtige Wegbereiterfunktion erfüllen: auf dem Gebiet der Entstehung und weiteren Stabilisierung einer „Medien-Moral “32 der Kinder und Jugendlichen und im Sinne der Schaffung eines kritischen, von Urteilskraft geprägten, ethischen Zugangs zu den vermittelten Werten durch Medien.

7. Schließlich muss es jedem Pädagogen bekannt sein, dass in seinem Blickfeld und pädagogischen Arbeitsgebiet „Kommunikation“ im Sinne von hermeneutischer Verständigung, Verbindung in Form eines interaktiven Diskurses, Lebensvorbereitung/-reform, medialer Decodierung sowie „Institutionalisierung“ einander bedingen. Wissenschaftliche Schulen und

\footnotetext{
${ }^{30}$ DiedeutscheErziehungsbewegung nahmbisin diedreißigerJahre sehr großen Einfluss auf die Entwicklung der Sowjetpädagogik. Diese starken reformpädagogischen Strömungen wurden bekanntlicherweise ebenso abrupt unter dem Joch der Stalin-Ära gestoppt wie die deutsche Erziehungsbewegung, beeinflusst durch die russischen Demokraten.

${ }^{31}$ Hofmann 1979, S. 32.

32 Arnold, B. Medienerziehung und moralische Entwicklung von Kindern. Frankfurt: Peter Lang, 1993, S. 208, s. auch S. 178 ff.
}

Forschungsstätten, Erziehungsvereine und eine vielgestaltige Landschaft pädagogischer Einrichtungen gewoben zu einem tragfähigen Netz - müssen Ziel und Bedingung der Bildungspolitik/-wissenschaft in einer pluralistischen Gesellschaft sein. ${ }^{33}$

Pädagogik als Erziehungswissenschaft darf daher den Zusammenhang zwischen Unterrichtsweise und persönlicher Einstellung von Lehrern und Schülern nicht außer Acht lassen.

\section{Reflexionen des Zusammenhangs von Un- terrichtsweise und persönlicher Einstellung von Lehrenden}

Auf dem Gebiet der Schulpädagogik kristallisierte sich im letzten Jahrzehnt unter anderem ein interessantes Anregungspotential für die Allgemeine Pädagogik heraus. Es geht um eine Bildungsauffassung, die den aktuellen Notwendigkeiten entspricht. Dieser liegt die vielgenannte Einheit von Bildung, Erziehung, Sozialisation und Enkulturation zugrunde. Es gibt in der Bildungsgeschichte viele nachvollziehbare Versuche, das Lernen zu beschleunigen und intensiver zu gestalten. In diesem Zusammenhang regte 1917 und besonders 1922 der Leipziger Reformpädagoge Hugo Gaudig (1860-1923) an, mit seiner Methode der „freien geistigen Arbeit“ („Tätigkeit"), den Schüler auf, eigene Wege mit eigenen Mitteln zu eigenen Zielen' zu führen - im Sinne der Selbstbestimmung bei Persönlichkeitsbildung $(1923)^{34}$

Erziehungsgeschichtlich ist dabei von Interesse, dass die Herbartianer wie Tuiskon Ziller (18171882) oder Wilhelm Rein (1847-1929), in denen sich die Herbartsche Tradition verkörpert hatte, in ihren Grundaussagen als eigentliche Wegbereiter der Reformpädagogik gelten, obwohl sie aus unterrichtsdidaktischer Sicht als Formalisierer Herbartscher Ideen firmieren. Bei T. Ziller sowie bei W. Rein sind schon ähnliche pädagogische Anschauungen wie bei $\mathrm{H}$. Gaudig entwickelt worden, und vom Prinzip der Individualität des Lernenden als auch vom Erziehungsverständnis im Hinblick auf Persönlichkeitsbildung zeigen sich fast übereinstimmende Vorgehenswei-

\footnotetext{
${ }^{33}$ Vgl. Hofmann 1979, S. 32.

${ }^{34}$ Gaudig, H. Die Schule im Dienste der werdenden Persönlichkeit. Erster Band. Leipzig: Quelle \& Meyer; 1917; Gaudig, H. Die Schule im Dienste der werdenden Persönlichkeit. Zweiter Band. Leipzig: Quelle \& Meyer; 1917; Gaudig, H. (Hrsg.) Freie geistige Schularbeit in Theorie und Praxis. Im Auftrage des Zentralinstituts für Erziehung und Unterricht. Breslau: Ferdinand Hirt, ${ }^{2}$ 1922; Gaudig, H. Die Idee der Persönlichkeit und ihre Bedeutung für die Pädagogik. Leipzig: Quelle \& Meyer, 1923.
} 
sen. ${ }^{35}$ So postuliert Ziller in seiner „Grundlegung zur Lehre vom erziehenden Unterricht“: „Dem Erziehungslehrer gegenüber und in der Mitte der Erziehungsanstalt ist der Lernende gegenüber immer ein ganzer Mensch ..., dessen Geistesfortschritte nicht abgesondert gedacht werden dürfen von seinen Fortschritten oder Rückschritten auf dem Wege zur Erfüllung seiner moralisch-religiösen Bestimmung. Als bloße Abrichtungsanstalt für ein Gewerbe oder zum Fortkommen darf sich eine Erziehungsschule nicht ansehen oder behandeln lassen [...]. Es kann auch da, wo der Begriff des erziehenden Unterrichts festgehalten wird, Niemand ein guter Lehrer sein, ohne daß er zugleich ein tüchtiger Erzieher wäre." ${ }^{\text {"6 }}$

Intensives Lernen und Bilden sind mit Prozessen der Persönlichkeitsentwicklung verbunden. Groß ist die Zahl pädagogischer und methodischer Arbeiten, die über Potenzen des Lehrstoffes geschrieben worden sind. Die vielgenannte Einheit aller Faktoren im Unterricht wurde oft durch die Steigerung des Bildungsgutes angestrebt. Selbst dort, wo Lehrende in Schule und Bildungseinrichtungen um eine Aktivierung dieser immanenten Erziehungs-, Bildungs- und Sozialisationspotenzen, die der Unterricht bietet, bemüht sind, zeigen sich Grenzen dieser vereinfachenden Auffassung. Unterrichten verlangt noch andere Bedingungen und Triebkräfte von starker sozialisierender Wirkung. Sie müssen zu den Erziehungspotenzen, die im wissenschaftlichen Inhalt des Unterrichtsstoffes ihre Quelle haben, hinzutreten und zu einer alters- und situationstypischen Einheit verschmelzen. Diese Einheit ist hauptsächlich durch eine aktive Grundeinstellung und eine zur Tätigkeit drängenden Grundhaltung im Unterricht und meist im gesamten schulischen Leben gekennzeichnet. Es genügt heute nicht mehr, nur von schöpferischer Tätigkeit und Selbsttätigkeit im Unterricht zu sprechen. Weit verbreitete Unzulänglichkeiten des Unterrichts, der nicht auf der Einheit von Bildung, Erziehung, Sozialisation und Enkulturation beruht, müssen uns bewusst werden.

Es soll im Folgenden zur Illustration der Aussagen der Versuch einer Zeichnung eines Gegenbildes von wissenschaftlichem Unterricht unternommen werden. Dieses Gegenbild zu allen historischen Erfahrungen und heutigen Erkenntnissen kennzeichnet

\footnotetext{
35 In der offiziell gewollten sowjetischen und auch der DDRPädagogik waren Herbartianer sogenannte "Prügel-“ oder „Rohrstockpädagogen“.

${ }^{36}$ Ziller, T. Grundlegung zur Lehre vom erziehenden Unterricht. Nach ihrer wissenschaftlichen und praktisch-reformatorischen Lehre entwickelt. Leipzig: Louis Pernitzsch, 1865, S. 20 (\$2. Doppelte Art des Unterrichts).

sich hauptsächlich durch unzureichende Mobilisierung von Einstellungen, die Grundlage des Aufbaues von Selbstkonzepten bei Schülerinnen und Schülern sind. Es handelt sich um genau das Gegenbild, wie es alle unter sowjetischem Einfluss stehenden Lehrenden praktizieren mussten. ${ }^{37}$

Typische Symptome dafür sind:

1. Sieht man von einer Reihe von Einzelmomenten $\mathrm{ab}$ und versucht das Typische aufzufinden, so kann man feststellen, dass der Akteur, also derjenige, der im Unterrichtsgeschehen eigentlich und hauptsächlich tätig wird, auch heute noch der Lehrende ist. Seine Bildungs- und Erziehungsfunktion im Unterricht besteht im überwiegenden Maße darin, den Stoff, das Bildungsgut, vorzutragen, darzustellen, zu erläutern, zu analysieren und zu begründen. Er fordert Verhaltensweisen und Bewusstsein und will Verhalten und Bewusstsein auf die Schüler übertragen. Aufs Ganze gesehen herrscht die darstellende, vortragende, vermittelnde und fordernde Lehr- und Erziehungsweise vor. Sie prägt als Fremdbestimmung den Charakter des Unterrichtsgeschehens. Der Lernende befindet sich vorwiegend in einer passiven Haltung. Er ist hauptsächlich Zuhörer und Zuschauer, er wird gefragt, ihm werden Tätigkeiten aufgetragen, er wird hinsichtlich seines Tuns überprüft. Er hört vornehmlich dem sprechenden Lehrenden zu, beobachtet dessen Versuche, verfolgt dessen Erklärungen, denkt auf Grund der Anweisungen mit, d.h. er ,folgt ' dem Unterricht. Der Lernende wird folgerichtig ungesprächig und sprachungewandt. Der Ausdruck verarmt, die Antworten schrumpfen auf einzelne Wörter zusammen. Die Verschlechterung der Schrift, die äußere Unordnung im Unterricht und bei der Führung der Hefte, alles hängt in gewissem Maße mit dieser soeben gekennzeichneten Situation zusammen. Schüler werden in Klischees gepresst, sie haben auswendig zu lernen.38 Selbständig entwickelnd, kreativ tätig werden in Richtung einer explorativen Pädagogik, dieser Prozess findet nicht statt. Perpetuiert wird eine ,Pädagogik des gehorsamen Schülers', eine Erziehung mit verhängnisvollem historischen Hintergrund. Wozu wir erziehen sollten, ist nicht der gehorsame, sondern gerade der aufgeweckte, mitdenkende, widersprechende, kämpfende, kritische Schüler.

\footnotetext{
${ }^{37}$ Allerdings war dies vielen Lehrern schon bewusst, und sie haben ständig Versuche unternommen, aus diesem Dilemma auszubrechen.

${ }^{38}$ Dieses Verfahren wurde bis zu den Examina der Studenten perpetuiert. Zu den Examensgebieten gab es Fragen, die auswendig gelernt und vorgetragen wurden.
} 
2. Ein zweites Kennzeichen des Gegenbildes bezieht sich auf die Art und Weise der Wissensvermittlung, wobei heutzutage vielerorts immer noch im Unterricht weitgehend die mündliche Methode der Wissensvermittlung vorherrscht. Das, Vermitteln des Stoffes', ein Terminus, der sich bezeichnenderweise gerade für diese Unterrichtsform eingebürgert hat, schafft allerdings nicht die besten Voraussetzungen für Aufmerksamkeit, Konzentration, Interesse und Lernbereitschaft, im Gegenteil. Der Stoff ist hierbei etwas, was sich sozusagen außerhalb des Schülers befindet und von ihm auf- bzw. angenommen werden soll. Der Lehrer ,übergibt' dem Schüler den Stoff oder das Wissen. In dieser typischen Situation kann die konzentrierte Aufmerksamkeit nur durch ständiges Ermahnen, Erinnern, Auffordern oder Zurechtweisen erhalten werden. Der Schüler ,fällt' allzu oft aus dem Unterricht, heraus', er schaltet ab und geht seinen eigenen Gedanken nach. Erst die Aufforderung zur Wiederholung, zur Übung, Zusammenfassung oder zur Leistungskontrolle veranlasst ihn wieder, konzentriert mitzuarbeiten.

3. Kriterium jedes Unterrichts ist die Erkenntnisaktivität des Lernenden. Sie weckt Freude an der Entdeckung, an der Lösung, an der Klärung von Problemen. Sie bewirkt die erregende Ungeduld und Neugier sowie Freude am Erkennen. Für das Gegenbild wissenschaftlichen Unterrichts ist die ungenügende Stimulierung der Erkenntnisaktivität typisch. Sie resultiert hauptsächlich daher, dass der Anstoß in den meisten Fällen von außen kommt. Der Schüler denkt, weil er dazu aufgefordert wird; über weite Strecken fehlen kraftvolle Antriebe. So ist in vielen Fällen festzustellen, dass keine wesentliche selbständige "geistige (denklogische) Arbeit" der Lernenden im Sinne Hugo Gaudigs zustande kommt, die in der Lage wäre, das Selbstkonzept auszubauen und zu fördern.

4. Das Gegenbild ,wissenschaftlichen', vom Lehrerethos geprägten ,modernen', handlungsorientierten Unterrichts ist weithin dadurch gekennzeichnet, dass der Lehrer in vielen Fällen nicht anzugeben vermag, wo der einzelne Schüler im Aneignungsprozess steht, welche Fehler er begangen hat, was er nicht verstanden hat. Erst bei einem ,durchdachten System der Leistungskontrolle kann er feststellen, was der Schüler weiß oder nicht weiß, wie sicher und vollständig sein Wissen und Können sind. Die Leistungskontrolle informiert aber nur über Resultate des vorausgegangenen Prozesses. Die Wissensaneignung, die Übung von Fähigkeiten und die Einübung von Fertigkeiten werden aber durch den herkömmlichen Wechsel von Lehrerdarstellung, Un- terrichtsgespräch und Stoffzusammenfassung nicht offenbar. Der Weg führt nicht zu einzelnen Schülern hin, sondern er weist zum anonymen Durchschnitt, zur Masse. Die Reformpädagogen haben mit der Methode eines ,freien, zum konkludierendem Denken führenden Arbeitens', versucht, dem Desinteresse der Lernenden entgegenzusteuern, auch in der Absicht, mit dieser Unterrichtskonzeption den Prinzipien der Selbsttätigkeit, Individualität und Anschaulichkeit Priorität einzuräumen, d.h. Schüler selbsttätig arbeiten zu lassen, diese Arbeitsvorgänge zu beobachten, anzustoßen, zu lenken, dem Schüler selbst die Arbeitsergebnisse vorlegen zu lassen, eigenen Willen zu unterstützen, dies ist die Alternative des Unterrichtens.

5. Die praktizierte Orientierung auf das Durchschnittstempo beim Lernen, das durch das Mittel der Klasse bestimmt wird, widerspricht der Auffassung von innovativem schüler- und im hier genannten Sinne, wissenschaftsorientiertem ' Unterricht. Auf die Folgen dieser durchschnittlichen Unterrichtsgeschwindigkeit - dieses durchgängige pädagogische Zentralproblem - ist in jeder geschichtlichen Periode hinreichend verwiesen worden. Dadurch, dass den leistungsstarken und leistungsbereiten Schülern zu wenig und den leistungsschwachen und antriebsärmeren zu viel Anregung abverlangt wird, entstehen Gefühle der Unausgefülltheit und bei anderen des Unberücksichtigtseins.

Auch dieser typische Zug des Unterrichts ruft in Monaten und Jahren merkliche Rückwirkungen hervor. Mit zunehmender Zeit bewirkt er eine Minderung der Aufmerksamkeit und der Lern- und Arbeitsbereitschaft dort, wo eine Erhöhung notwendig wäre. Zwischen diesen Einstellungen zum Unterricht und allgemeiner Interessiertheit bestehen auch $\mathrm{Zu}$ sammenhänge zur Inaktivität der Schüler. Es ist ein Problem des (praktischen) Erkenntnisinteresses, das hier angesprochen wird.

6. Alle bisher genannten Kennzeichen machen insgesamt eine Erstarrung des pädagogischen Denkens sichtbar, die man auch Formalisierung nennen könnte. Monotonie und Stereotypie des Unterrichtsverlaufs wirken zusammen mit den bereits genannten Erscheinungen nachteilig auf die Lerneinstellung der Schüler. Sehr bald kann sich, wenn die ersten Schuljahre vorüber sind, ein gewisses Gefühl der Einförmigkeit beim Schüler entwickeln, das sich mitunter zu Unlust und Abneigung gegenüber der Schule und dem Lernen ausweitet. Und damit beschreiben wir wiederum die Kontaktstelle zwischen Unterrichtseinstellung und Aufgeschlossenheit für Ideale und Normen. 
7. Sogenannte Stoffvermittlungsmethoden nutzen überhaupt nichts, wenn der Unterricht nicht als „Erziehender Unterricht" angelegt ist. Im 16. Absatz seiner Einleitung zur „Allgemeine(n) Pädagogik aus dem Zweck der Erziehung abgeleitet" (1806) formuliert Herbart:

„Pädagogik ist die Wissenschaft, deren der Erzieher für sich bedarf. Aber er soll auch Wissenschaft besitzen zum Mitteilen. Und ich gestehe gleich hier, keinen Begriff zu haben von Erziehung ohne Unterricht; so wie ich rückwärts, in dieser Schrift wenigstens, keinen Unterricht anerkenne, der nicht erzieht. Welche Künste und Geschicklichkeiten ein junger Mensch um des bloßen Vorteils willen von irgendeinem Lehrmeister lernen möge, ist dem Erzieher an sich ebenso gleichgültig, als welche Farbe er zum Kleide wähle. Aber wie sein Gedankenkreis sich bestimme, das ist dem Erzieher alles; denn aus Gedanken werden Empfindungen, und daraus Grundsätze und Handlungsweisen. ${ }^{\text {" }} 9$

Das Gegenbild wissenschaftsorientierten, vom Lehrerethos geprägten ,modernen', handlungsorientierten Unterrichtens ist an seiner geringen erzieherischen Wirkung zu erkennen. Unterricht dieser Methodik leistet vor allem der Disziplinlosigkeit Vorschub $^{40}$, und er schafft gleichwohl potentielle Voraussetzungen dafür, dass beispielshalber Unordnung und Desinteresse Platz greifen können, wenn andere Anlässe innerhalb der Faktorenkomplexion hinzukommen. Vielfach gilt als Ursache für Lernunlust, allgemeine Interesselosigkeit und Disziplinlosigkeit die Unterdrückung des Drangs nach Selbsttätigkeit; das Selbstbewusstsein und der Leistungswille, die bei jungen Menschen stark ausgeprägt sind, werden verletzt und gemindert, und es werden zu wenig explorierende Erlebnisse eigener Leistung hervorgebracht.

\section{Lösungsmöglichkeiten}

Diese genannten und noch eine Reihe nicht genannter Merkmale kennzeichnen ein Gegenbild von Unterricht. Ein sich davon in jeder einzelnen Position abhebender so genannter, wissenschaftlich geprägter, aus dem Lehrerethos, aus der Verpflichtung und Verantwortung dem Kinde gegenüber, in der Auffassung von ,Führen im Sinne von Leiten und ,Wachsenlassen' begründeter, wachsender ,re-

\footnotetext{
${ }^{39}$ Herbart, J.F., ebd., 1976, S. 76.

${ }^{40}$ Natürlich hat Disziplinlosigkeit noch viele andere Ursachen, denn Unterricht definiert sich nach Friedrich Winnefeld (1911-1968) u. Mitarbeitern als „Faktorenkomplexion“ (Forschungsbericht 1956; Publikation 1957; ${ }^{2} 1963$ u. ff.), und somit ist Disziplin immer als Ergebnis eines Gesamtprozesses zu betrachten.

flexiv-moderner' handlungsorientierter Unterricht, der ,kindgemäße Erziehung' intendiert, sollte Lehrende und Lernende im Hinblick auf ihre Selbstverwirklichung nach eigenen Interessen auf eigenen Wegen mit eigenen Zielen suchen lassen.

Bekannterweise nennt Theodor Litt seine 1927 erschienene Schrift „,Führen” oder, Wachsenlassen “41. Auf den beiden letzten Seiten seines Schlusskapitels formuliert er:

"(B)eide Begriffe bezeichnen zunächst nichts weiter als Gleichnisse, die das Wesen des erzieherischen Tuns schnell und eindrucksvoll vor das innere Auge stellen sollen. Aber es gibt kein Gleichnis, das nicht das in ihm dargestellte in die Gefahr brächte, hinter dem Bilde zu verschwinden. Solches aber geschieht in unserem Falle am unfehlbarsten immer dann, wenn eines dieser Bilder, vom Gegenbilde abgelöst, den ganzen Sinn der Erziehung in sich auszusprechen beansprucht. Denn damit wird dasjenige Moment der Erziehung, dem dieses Bild zugeordnet ist, aus dem gegensatzreichen Gefüge herausgerissen, innerhalb dessen es einzig seine Bestimmung erfüllen kann; es setzt sich absolut, d.h. dem Ganzen der Erziehung gleich, und hebt damit deren Sinn in Wahrheit auf. Von solcher Selbstübersteigerung kann es sich nur dadurch schützen, dass es sich allezeit an seinem Gegenbild berichtigt und begrenzt. ${ }^{\text {“42 }}$

Litts Gedanken kulminieren in der Feststellung:

„In verantwortungsbewußtem Führen niemals das Recht vergessen, das dem aus eigenem Grunde wachsenden Leben zusteht - in ehrfürchtig-geduldigem Wachsenlassen niemals die Pflicht vergessen, in der der Sinn erzieherischen Tuns sich gründet - das ist der pädagogischen Weisheit letzter Schluß “ ${ }^{43}$

Geht man vom Problem der Selbstverwirklichung aus, so ist noch eine weitere Seite zu berücksichtigen: dass sich Lehrende und Lernende für Werte und Normen der Gemeinschaft öffnen, da sonst eine Entwicklung eintreten würde, die genauso verhängnisvoll wäre wie das Diktatorische, Doktrinäre der Vergangenheit. Es ist bedenklich, den Lehrenden als absolut zu setzen, den Lernenden i.w.S. zu indoktrinieren, ihm nicht die Möglichkeit einzuräumen, das Lehrerverhalten und -handeln zur Disposition stellen zu können, oder im anderen Extrem: es dem Schüler allein zu überlassen, wie er seinen zukünftigen Lebensweg zu beschreiten hat.

\footnotetext{
${ }^{41}$ Litt, Th. "Führen“ oder „Wachsenlassen“. Eine Erörterung des Pädagogischen Grundproblems. Leipzig/ Berlin: B. Teubner, 1927.

${ }^{42}$ Ebd., S. 99 f.

${ }^{43}$ Ebd., S. 100.
} 
Die Schulwirklichkeit zeigte und zeigt, dass mangelhaft ausgeprägte Emotionalität, Willens- und Kräftepotentiale, Motivationsbereitschaft oder auch Interessen Ursachen und Folgen des Unbehagens im Schulalltag sind.

Daraus resultieren folgende Thesen

„Bildungsprozesse sind viel stärker als Selbstbildungsprozesse zu entwerfen. Sie brauchen Spielraum und vielfältige Angebote, die eine sinnorientierte selbstbestimmte Aneignung des Lehrerberufs ermöglichen. Forschung und Lehre muss effektiver und dauerhaft miteinander verbunden werden." ${ }^{4}$

„Besagte Ausbildung - und damit verbundene Bildung - muss die vorhandene Spannung zwischen wissenschaftlichem Reflektieren der Berufspraxis bzw. Professionalisierung und dem Erwerb von Berufskompetenzen erkennen, analysieren, strukturieren und effektiv in Handlungskompetenzen umsetzen. " ${ }^{\text {"5 }}$

\section{gen}

\section{„3. Grundstein für lebenslanges Lernen le-}

- Lehrerbildung und -ausbildung muss heutzutage vermitteln, dass die persönliche Entwicklung und Befähigung, die Ausbildung für das Lehramt niemals abgeschlossen, ja ein lebenslanger Lernprozess ist. Eine Abkehr von der Ausbildungsfixierung bedeutet, die Studierenden müssen wesentliche Lernbausteine kennen lernen, mit denen sie in Zukunft professionell berufliche Probleme angehen können.

- Dazu zählen:

- die Fähigkeit zur Selbstkritik, verbunden mit dem ständigen Erwerb und Ausbau von Selbst- und Sozialkompetenz;

- die methodische Offenheit, die befähigt, auf unterschiedliche Lerngruppen differenziert (anders) zu reagieren und sich stets neu Anregungen zu holen;

- die Fähigkeit zur Analyse von Lerntypen (und -typologien) und entsprechenden Lernwegen, sich selbst eingeschlossen;

- im Team arbeiten, planen, durchführen und evaluieren zu wollen und zu können;

- die Fähigkeit, aktuelle Diskussionen in existierende oder neu zu schaffende pädagogische Denkmodelle einordnen und hinsichtlich ihrer sich wandelnden norma-

\footnotetext{
${ }^{44}$ Hoffmann, K., Reinert von Carlsburg, G.-B. Lernen in der Informationsgesellschaft - ein Leben lang. In: Towards the Learning Society. Hrsg. v. Jucevičienè, P., Merkys, G., Reinert von Carlsburg, G.-B. Frankfurt a.M. u.a.: Peter Lang, 2002, S. 329.

${ }^{45}$ Ebd., S. 331.
}

tiven Konsequenzen für die Praxis bewerten zu können." ${ }^{\text {"46 }}$

\section{Literatur}

1. Arnhardt, G. \& Reinert [v. Carlsburg], G.-B. (Hrsg.) (1996). Jan Amos Comenius. Über sich und die Erneuerung von Wissenschaft, Erziehung und christlicher Lebensordnung. Bände 1 u. 2. Donauwörth: Auer Verlag.

2. Arnold, B. (1993). Medienerziehung und moralische Entwicklung von Kindern. (S. 178 ff.). Frankfurt: Verlag Peter Lang.

3. Belinski, W. G. (1953). Ausgewählte pädagogische Schriften. Berlin: Volk u. Wissen.

4. Benner, D. (Hrsg.) (1997). Johann Friedrich Herbart. Systematische Pädagogik. Bände $1 \mathrm{u}$. 2. Weinheim: Deutscher Studien Verlag.

5. Brockhaus' Konversations-Lexikon (1894a). Band 3. (14. Auflage). Leipzig/Berlin/Wien: Brockhaus Verlag.

6. Brockhaus' Konversations-Lexikon (1894b). Band 5. (14. Auflage). Leipzig/Berlin/Wien: Brockhaus Verlag.

7. Brockhaus' Konversations-Lexikon (1894c). Band 9. (14. Auflage). Leipzig/Berlin/Wien: Brockhaus Verlag.

8. Brockhaus' Konversations-Lexikon (1895). Band 15. (14. Auflage). Leipzig/Berlin/Wien: Brockhaus Verlag.

9. Diesterweg, A. (1820). Ueber Erziehung im Allgemeinen und Schulerziehung im besonderen. Elberfeld: Buschler.

10. Diesterwegs, F. A. W. (Bearb. u. Hrsg.) (1835). Wegweiser zur Bildung für Lehrer und die Lehrer werden wollen, und methodisch-praktische Anweisungen zur Führung des Lehramtes. In Gemeinschaft mit K. Bormann, E.J. Hentschel, A. Lüben, Mädler \& F. Schubart. 2 Bde. Essen: G. D. Bädeker [Baedeker].

11. Diesterweg, F. A. W. (Bearb. u. Hrsg.) ([2] 1838; fortgef. u. verm. Aufl. ${ }^{3} 1844$; verb. Aufl. $\left.{ }^{4} 1850\right)$. Wegweiser zur Bildung für deutsche Lehrer. In Gemeinschaft mit K. Bormann u.a., Neue Aufl. in zwei Bänden. G. D. Bädeker [Baedeker].

12. Dobroljubow, N. A.(1956). Ausgewählte pädagogische Schriften. Berlin: Volk u. Wissen.

13. Gaudig, H. (1917). Die Schule im Dienste der werdenden Persönlichkeit. Erster Band. Leipzig: Quelle \& Meyer.

\footnotetext{
${ }^{46}$ Ebda., S. 334.
} 
14. Gaudig, H. (1917). Die Schule im Dienste der werdenden Persönlichkeit. Zweiter Band. Leipzig: Quelle \& Meyer.

15. Gaudig, H. (Hrsg.) (1922). Freie geistige Schularbeit in Theorie und Praxis. Im Auftrage des Zentralinstituts für Erziehung und Unterricht. (2. Auflage). Breslau: Ferdinand Hirt.

16. Gaudig, H. (1923). Die Idee der Persönlichkeit und ihre Bedeutung für die Pädagogik. Leipzig: Quelle \& Meyer.

17. Herbart, J. F. Systematische Pädagogik. Eingel., ausgew. u. interpr. v. Benner, D. Stuttgart: Klett-Cotta, 1986, S. 71-191

18. Hofmann, F. (1979). Probleme der „Allgemeinen Pädagogik" als Aufgabe der pädagogischen Grundlagenforschung. In F. Hofmann \& Ch. Fritzke (Hrsg.), „Allgemeine Pädagogik“ als Anliegen pädagogischer Grundlagenforschung. Kongreß- und Tagungsberichte (KTB) der Martin-Luther-Universität Halle-Wittenberg (MLU), Wissenschaftliche Beiträge 26 (E 23) (S. 5-33). Halle: MLU.

19. Hofmann, F. (1980). Zum Erkenntniswert der Wissenschaftsgeschichte der Pädagogik. In F. Hofmann (Hrsg.), Erkenntniswert einer Wissenschaftsgeschichte der Pädagogik. Kongreß- und Tagungsberichte (KTB) der Martin-Luther-Universität Halle-Wittenberg (MLU), Wissenschaftliche Beiträge 13 (E 28) (S. 5-47). Halle: MLU.

20. Hofmann, F. (1985). Wissenschaftssprache der Pädagogik in historischer Sicht. In F. Hofmann (Hrsg.), Die Wissenschaftssprache der Pädagogik. Problemlogische und -historische Beiträge. Kongreß- und Tagungsberichte (KTB) der Martin-Luther-Universität Halle-Wittenberg (MLU), Wissenschaftliche Beiträge 22 (E 65) (S. 5-20). Halle: MLU.

21. Hofmann, F. (1989). Studien zur Geschichte der bürgerlichen Didaktik. Berlin: Volk u. Wissen.

22. Hoffmann, K./Reinert von Carlsburg, G.-B. (2002). Lernen in der Informationsgesellschaft - ein Leben lang. In P. Jucevičienè/G. Merkys/ G.-B. Reinert (Hrsg.), Towards the Learning Society. (S. 327-340). Frankfurt: Verlag Peter Lang.

23. Kegler, D. (1991). Lev Tolstoj: Ethizistische Pädagogik. In D. Kegler, Das Ethos der russischen Pädagogik. Studien zum Erziehungsbegriff seit
Pirogov (S. 82-90). St. Augustin: Academia Verlag.

24. Kegler, D. (1991). Das Ethos der russischen Pädagogik. Studien zum Erziehungsbegriff seit Pirogov. St. Augustin: Academia Verlag.

25. Herbart, J. F. (1976). Ausgewählte Schriften zur Pädagogik. Ausgewählt, eingeleitet und erläutert von F. Hofmann unter Mitarbeit von B. Ebert (S. 67 ff.). Berlin: Volk u. Wissen.

26. Herder, J.G. (1952). Zur Philosophie der Geschichte. Eine Auswahl in zwei Bänden. Zweiter Band. Hrsg. von W. Harich. Reihe: Klassisches Erbe aus Philosophie und Geschichte. Berlin: Aufbau Verlag.

27. Herders Werke in fünf Bänden (1978). Bibliothek Deutscher Klassiker. Band 4 (Slawische Völker. 16. Buch. Kap. IV, S. 393-400). Hrsg. von den Nationalen Forschungs- u. Gedenkstätten der klassischen deutschen Literatur in Weimar. Ausgewählt u. eingeleitet von R. Otto. (5. Auflage). Berlin/Weimar: Aufbau-Verlag.

28. Litt, Th. (1927). „Führen“ oder „Wachsenlassen“. Eine Erörterung des Pädagogischen Grundproblems. Leipzig/Berlin: B. Teubner Verlag.

29. Petersen, P. (1927). Der Jena-Plan einer freien allgemeinen Volksschule. Langensalza: Julius Beltz.

30. Petersen, P. (Hrsg.) (1934). Die Praxis der Schulen nach dem Jena-Plan. In P. Petersen (Hrsg.), Eine freie allgemeine Volksschule nach den Grundsätzen Neuer Erziehung (Der Jena=Plan), Dritter Band. Weimar: Verlag Hermann Böhlaus Nachf.

31. Winnefeld, F. (1970). Pädagogisches Feld als Faktorenkomplexion. In: G. Dohmen, F. Maurer \& W. Popp (Hrsg.). Unterrichtsforschung und didaktische Theorie (S. 35-39). München: R. Piper.

32. Winnefeld, F. u. Mitarb. $\left({ }^{2} 1963 ;{ }^{3} 1965 ;{ }^{4} 1967\right.$; ${ }^{5}$ 1971). Pädagogischer Kontakt und pädagogisches Feld (S. 34-37). München/Basel: Reinhardt [1957 In Erziehung und Psychologie. Bd. 7 (S. 127-160). Beiheft der Zeitschrift Schule und Psychologie].

33. Ziller, T. (1865). Grundlegung zur Lehre vom erziehenden Unterricht. Nach ihrer wissenschaftlichen und praktisch-reformatorischen Lehre entwickelt. Leipzig: Louis Pernitzsch. 


\section{Santrauka}

\section{Gerd-Bodo von Carlsburgas}

\section{APIE NAUJOS PEDAGOGINIO MOKSLO ORIENTACIJOS PLĖTRA VIDURIO IR RYTU EUROPOJE BEI JOS İGYVENDINIMĄ PEDAGOGINĖJE PRAKTIKOJE}

Pirmoje straipsnio dalyje aptariama pažangios pedagoginès naujovès ir pasikeitimai, kurie tik tuomet gali būti įtikinami, jei yra paremti patikima argumentacija ir legitimacija. Mes turime užkirsti kelią tam, kad pedagogikoje madingos mintys ne- būtų išaukštinamos iki idejų arba net fikcijų, nes logiškos viso to pasekmès, kaip jau pastebėjo Franzas Hofmannas (žr. 2 išnašą / Hofmann 1979, p. 5-33, ypač p. 5), yra greičiau struktūrų ir terminų painiava. Mokslo istorijos duomenys yra pagrindas mąstyti.

Antroje dalyje aptariamos praktinès pasekmès, pamokos metodų ir asmeninio mokytojo nusistatymo sąryšis bei su tuo susijęs poveikis mokymo aplinkai. Taip pat minimi esminiai šiuolaikinio darbo trūkumai, be to, bandoma tradicinei, probleminei pamokos sampratai priešpriešinti pamoką, kuri remiasi mokytojo etika, istoriškai pagrịstomis ir laiko dvasiai artimomis dalykinèmis ir gebëjimu kompetencijomis.

Esminiai žodžiai: ugdymo procesas, mokslo istorija, mokymasis visą gyvenimą, profesinè kompetencija.

Heidelbergo edukologijos universitetas Iteikta $2011 \mathrm{~m}$. birželio men. 